\title{
Electrolyte Imbalance and Its Effect on QTC Interval in Patients Hospitalized with COVID-19
}

\author{
Murat Çap ${ }^{1},[M D]$ \\ ORCID: 0000-0003-1164-2124 \\ Bernas Altıntaş', [MD] \\ ORCID: 0000-0003-1662-3061 \\ Önder Bilge', [MD] \\ ORCID: 0000-0001-8214-972X \\ Ferhat Işık', [MD] \\ ORCID: 0000-0002-1438-3327 \\ Ümit İnci', [MD] \\ ORCID: 0000-0002-7412-1222 \\ Rojhat Altındağ', [MD] \\ ORCID: 0000-0002-2479-9318 \\ Abdurrahman Akyüz', [MD] \\ ORCID: 0000-0003-2491-6593 \\ Muhammed Süleymanoğlu', [MD] \\ ORCID: 0000-0003-1128-9455 \\ Burhan Aslan', [MD] \\ ORCID: 0000-0002-8994-7414 \\ ilyas Kaya', [MD] \\ ORCID: 0000-0003-1998-6722 \\ Mehmet Şahin Adıyaman', [MD] \\ ORCID: 0000-0003-2817-0629 \\ Derya Deniz Altıntaş², [MD] \\ ORCID: 0000-0002-2618-9177 \\ Neşe Kanbal ${ }^{3}$, [MD] \\ ORCID: 0000-0001-6560-5746 \\ Erkan Baysal', [MD] \\ ORCID: 0000-0001-6034-9413
}

\footnotetext{
'Department of Cardiology, SBU Diyarbakır Gazi Yaşargil Education and Research Hospital, Diyarbakır, Turkey.

2Department of Radiology, SBU Diyarbakır Gazi Yaşargil Education and Research Hospital, Diyarbakır, Turkey.

${ }^{3}$ Department of Internal Medicine, Dicle University Faculty of Medicine, Diyarbakır, Turkey.
}

\section{Corresponding Author: Murat Çap}

Department of Cardiology, SBU Diyarbakır Gazi Yaşargil Education and Research Hospital, Diyarbakır, Turkey.

Phone: +90 4122580060

E-mail: murat00418@hotmail.com

\section{n ABSTRACT Con}

Objective: Studies have shown that electrolyte abnormalities can be seen in Coronavirus disease 2019 (COVID-19) patients and are associated with the severity of the disease. We aimed to investigate the electrolyte imbalance such as hypocalcemia and hypokalemia in patients hospitalized with COVID-19 and its effect on the QTc interval.

Materials and Methods: 185 hospitalized patients with COVID-19 whose diagnosis was confirmed by a real-time reverse transcriptasepolymerase chain reaction and/or Computed Tomography of the chest were included in the study. The potassium and calcium measurements on the admission and third day of hospitalization were recorded. The QTc interval measurement was performed on the admission (QTC-B) and the 3rd-day (QTC-AT) electrocardiography.

Results: The median age was 43 (31-56 IQR) and 102 (55.1\%) of patients were male. Median baseline potassium level was $4.0 \mathrm{mEq} / \mathrm{L}$ (3.7-4.3 IQR) and corrected calcium level was $8.48 \mathrm{mg} / \mathrm{dl}$ (8.24-8.73 IQR), whereas the $3 \mathrm{rd}$-day median potassium level was $4.2 \mathrm{mEq} / \mathrm{L}$ (3.9-4.5 IQR) and corrected calcium level was $8.42 \mathrm{mg} / \mathrm{dl}$ (8.16-8.70 IQR).The median QTc-B interval was found as $427 \mathrm{~ms}$ (409-447 IQR) whereas the median QTC-AT interval was found as $438 \mathrm{~ms}$ (414-459 IQR). Median $9 \mathrm{~ms}$ (-5- 28 IQR) prolongation was observed in the QTC interval, which was statistically significant $(p<0.001)$. There was a significant relationship between QTc-AT and baseline potassium level, potassium level on the 3rd day (respectively $\beta$ coefficient $=-2.083,95 \% \mathrm{Cl}-27.134--0.577, \mathrm{p}$ value $=$ $0.041, \beta$ coefficient $=-2.547,95 \% \mathrm{Cl}-27.925--3.385, p$ value $=0,013$ ) but there was no significant relationship with baseline and 3rd day calcium level. (respectively $\beta$ coefficient $=-0.848,95 \% \mathrm{Cl}-22.102-8.919$, $\mathrm{p}$ value $=$ $0.399, \beta$ coefficient $=0.707,95 \% \mathrm{Cl}-8.685-20.784$, $p$ value $=0.415$ )

Conclusion: Electrolyte disorders such as hypocalcemia and hypokalemia can be seen in patients hospitalized with COVID-19. After treatment, a significant prolongation was observed in the QTc interval and a significant relationship was observed between QTC-AT and potassium levels, but no significant relationship with calcium was observed.

Keywords: COVID-19, QTc interval, Hypokalemia, Hypocalcemia 


\section{INRODUCTION}

Coronavirus disease 2019 (COVID-19) is caused by the recently emerged coronavirus, Severe acute respiratory syndrome coronavirus-2 (SARS-CoV-2), which was first reported in December 2019 in China [1]. The World Health Organization declared the COVID-19 as a Public Health Emergency in January 2020, and a pandemic on March 2020 [2, 3]. Although its primary target is the lung, it can also cause pathological changes in many organs and tissues. Some of its undesirable effects can be seen in the cardiovascular system as myocardial injury, myocarditis, acute coronary syndrome, and arrhythmias [4].

The QT interval reflects the duration of ventricular electrical activity determined by the phases of depolarisation and repolarisation. Several previous studies have shown the association between heart rate corrected QT (QTC) interval and cardiovascular mortality or all-cause mortality [5-7]. Hydroxychloroquine and azithromycin, which are used in the treatment of COVID-19, are drugs that have the potential to prolong the QT interval and cause torsade de pointes [8-10]. Due to both direct effects of COVID-19 on the cardiovascular system and the arrhythmic effects of hydroxychloroquine, azithromycin, and antiviral drugs used in the treatment, the prolongation of QTc interval could be seen in patients with COVID-19 during follow up.

In early COVID-19 studies, it has been stated out that some serum electrolyte disturbances may occur regarding sodium, potassium, and calcium [11, 12]. Especially hypokalemia was observed due to SARS CoV 2 interaction with the renin angiotensin aldosterone system [13, 14]. Hypokalemia and hypocalcemia may result in life threatening ventricular arrhythmias due to prolonged QTc, in patients with underlying cardiac disease or patients using drugs that prolong the QTc interval $[15,16]$. In this study, We aimed to investigate the electrolyte imbalance such as hypocalcemia and hypokalemia in patients hospitalized with COVID-19 and its effect on the QTc interval.

\section{MATERIALS and METHODS}

This is a single-center retrospective observational study. Patients hospitalized with COVID-19 in our hospital between April 6 and May 5, 2020, were included in the study. An identified case of COVID-19 was defined as a positive finding on real-time reverse transcriptase-polymerase chain reaction (RT-PCR) assay of nasal and pharyngeal swab specimens and/or typical finding of COVID-19 on Computed tomography (CT) of the chest. The demographic, clinical characteristics, and laboratory parameters of patients during hospitalization were collected from the electronic medical records of the hospital.

Admission to the emergency department was accepted as the 1st day, and the electrocardiography (ECG) was recorded in the first and third days of hospitalization. The patients who had no RT-PCR positivity and typical findings in chest $C T$, who did not have ECG records and who we could not start hydroxychloroquine or hydroxychloroquine plus azithromycin due to basal prolonged QTc interval or any other reason, who had atrial fibrillation and permanent pacemakers, who had missing potassium and calcium measurement were excluded from the study. A total of 230 consecutive patients were screened for the study and 185 patients were included in the study after the exclusion criteria.

The potassium and calcium measurements on the admission and third day of hospitalization were recorded. Approximately $\% 50$ of calcium in serum is bound to proteins, principally albumin. Albumin is a negative acute phase reactant and its levels may decrease due to infection, parallel to this calcium levels can be measured low [17]. Therefore, corrected calcium level was calculated as shown in equation (corrected calcium $(\mathrm{mg} /$ $\mathrm{dL})=$ total calcium $(\mathrm{mg} / \mathrm{dL})+0.08 \times(40$-albumin $(\mathrm{g} / \mathrm{L})$ ) [18]. Calcium level $<8.5 \mathrm{mg} / \mathrm{dL}$ was defined as hypocalcemia and potassium level $<3.5 \mathrm{mEq} / \mathrm{L}$ was defined as hypokalemia $[19,20]$.

Myocardial injury was defined by the presence of at least one cardiac troponin value above the 99th percentile upper reference limit. Four systemic inflammatory response syndrome (SIRS) criteria were defined, tachypnea (respiratory rate $>20$ breaths/min), namely tachycardia (heart rate $>90$ beats/min) fever or hypothermia (temperature $>38$ or $<36{ }^{\circ} \mathrm{C}$ ), and leukocytosis, leukopenia, or bandemia (white blood cells $>12,000 / \mathrm{mm} 3,<4,000 /$ $\mathrm{mm} 3$ or bandemia $\geq 10 \%$ ). SIRS was accepted as 
an occurrence of two or more than two of these criteria.

Hydroxychloroquine was given orally at $400 \mathrm{mg}$ BID for the first day (loading dose) followed by $200 \mathrm{mg}$ BID for 4 days. Azithromycin was given orally at a dose of $500 \mathrm{mg}$ for one day (loading dose) followed by $250 \mathrm{mg}$ daily for 4 days.

\section{QTc Interval Measurement}

All standard 12-lead ECGs were recorded at $25 \mathrm{~mm} / \mathrm{s}$ speed and $10 \mathrm{~mm} / \mathrm{mv}$ gain. The QT interval measurement was performed manually by 2 experienced cardiologists on the admission (baseline QTc(QTc-B)) and 3rd-day ECG (QTc after treatment (QTc-AT)). The QT interval was measured from the beginning of the QRS complex to T wave termination and averaged over 3 to 5 beats. In cases where the T-wave was interrupted by a U-wave, the end of the T-wave was defined as the nadir between the T- and U-waves. QT intervals were corrected for the effects of heart rate using a modified Bazzet's formula $(\mathrm{QTC}=\mathrm{QT} /(\mathrm{R}-\mathrm{R}) 1 / 2)$.

The study was approved by the ministry of health and the local institutional ethics committee. The study protocol conforms to the Declaration of Helsinki.

\section{Statistical Analysis}

Continuous variables are presented given as median interquartile range $25-75 \%$ (IQR) due to non-normal distribution. Categorical variables were expressed as percentages. Wilcoxon rank test was used to assess the change of QTC after treatment. The univariable and multivariable linear regression analysis was used to assess the relationship of QTCAT with calcium and potassium. We constructed two separate models to assess the relationship of QTc-AT with baseline and 3rd-day potassium and calcium levels after adjusting common clinical and biological plausible predictors ( age, gender, QTC prolonged drug(azithromycin, antiviral treatment), loop diuretic, beta-blocker, congestive heart failure(CHF), chronic renal failure (CRF), SIRS criteria $\geq 2$, myocardial injury, magnesium). In all the statistical analyses, a $P$ value of $<0.05$ is considered statistically significant. The data were analyzed using SPSS (Statistical Package for Social Science) 24.0.

\section{RESULTS}

185 COVID-19 patients whose diagnosis was confirmed by RT-PCR and/or Chest CT were included in our study. The median age was 43 (3156 IQR) and 102 (55.1\%) of patients were male. Baseline and clinical characteristics were presented in Table 1.

Radiographic findings of pneumonia were observed in 150 (81\%) patients. 28 (15,1\%) patients had hypertension, 27 (14.6\%) patients had diabetes mellitus, and $12(6.5 \%)$ patients had chronic respiratory disease. 11 (6\%) patients had coronary artery disease, 6 (3.2\%) patients had $\mathrm{CHF}$, and $5(2.7 \%)$ patients had the myocardial injury. SIRS criteria $\geq 2$ was observed in 40 (21.6\%) patients. All patients received hydroxychloroquine,

Table 1. Baseline and clinical charecteristics of patients

\begin{tabular}{|l|c|}
\hline & $\mathrm{n}=185$ \\
\hline Age(year) & $43(31-56)$ \\
\hline Gender(male) & $102(55.1 \%)$ \\
\hline Hypertension & $28(15.1 \%)$ \\
\hline Diabetes mellitus & $27(14.6 \%)$ \\
\hline Smoking & $58(28.6 \%)$ \\
\hline Congestive heart failure & $6(3.2 \%)$ \\
\hline Coronary artery disease & $11(6 \%)$ \\
\hline Chronic respiratory disease & $12(6.5 \%)$ \\
\hline Chronic renal failure & $5(2.7 \%)$ \\
\hline Myocardial injury & $5(2.7 \%)$ \\
\hline$\geq 2$ SIRS criteria & $40(21.6 \%)$ \\
\hline Radiographic finding of pneumonia & $150(81 \%)$ \\
\hline Lenght of stay hospital (day) & $6(5-8)$ \\
\hline Invasive mechanical ventilation & $13(7 \%)$ \\
İntensive care unit admission & $25(13.5 \%)$ \\
\hline Loop diuretic & $5(2.7 \%)$ \\
\hline Beta blocker & $16(8.6 \%)$ \\
\hline ACEl/ARB & $15(\% 8.1)$ \\
Hydroxychloroquine & $185(\% 100)$ \\
\hline Azithromycine & $111(\% 60.9)$ \\
\hline Favipiravir & $23(\% 12.4)$ \\
\hline Antibiotics & $67(36 \%)$ \\
\hline Potassium replacement & $17(9.1 \%)$ \\
\hline Calcium replacement & $9(4.9 \%)$ \\
\hline Temperature ( $\left.{ }^{\circ} \mathrm{C}\right)$ & $37.2(36.8-37.7)$ \\
\hline Systolic blood pressure (mmHg) & $110(100-120)$ \\
\hline Diastolic blood pressure (mmHg) & $70(60-80)$ \\
\hline
\end{tabular}

Continuous variables are presented given as median (interquartile range) and categorical variables were expressed as number (\%). SIRS, Systemic Inflammatory Response Syndrome 
$112(60.5 \%)$ patients received azithromycin, and 23 (12.4\%) patients received favipiravir. The median hospitalization period was 6 days ( 5-8 IQR).

$25(13,5 \%)$ patients were admitted to the intensive care unit and $13(7 \%)$ patients were mechanically ventilated.Ventricular arrhythmia was not observed, and there were no arrhythmic deaths; however, 11 (\%6) of the patients died from respiratory failure and/or sepsis in the intensive care unit follow-up.

Median baseline potassium level was $4.0 \mathrm{mmol} / \mathrm{L}$ (3.7-4.3 IQR), and corrected calcium level was $8.48 \mathrm{mg} / \mathrm{dl}$ (8.24-8.73 IQR), whereas the 3rd-day median potassium level was $4,2 \mathrm{mmol} / \mathrm{L}(3,9-4,5$ IQR), and corrected calcium level was calculated as $8.42 \mathrm{mg} / \mathrm{dl}$ (8.16- 8.70 IQR). Potassium $<3.5$ $\mathrm{mEq} / \mathrm{L}$ was observed in $16(8.6 \%)$ patients and the number of patients with $\leq 4 \mathrm{mEq} / \mathrm{L}$ was 99 (53.5\%). Hypocalcemia (corrected calcium $<8.5$ $\mathrm{mg} / \mathrm{dl}$ ) was observed in 95 (51\%) patients when the correction was applied to all patients whereas hypocalcemia was observed in 44 (24\%) of patients when the correction was applied only to those with albumin below $40 \mathrm{mg} / \mathrm{L}$. It was determined that 17 (9.2\%) patients had potassium and 9 (4.9\%) patients had calcium replacement. Laboratory and electrocardiographic parameters were given in Table 2.

The median QTc-B interval was found as 427 ms (409447 IQR) whereas the median QTc-AT interval was found as 438 ms (414-459 IQR). Median 9 ms (-5-28 IQR) prolongation was observed in the QTc interval, which was statistically significant $(p<0.001)$. After treatment, 11 patients had QTc $\geq 500 \mathrm{~ms}$ and/or $\Delta \mathrm{QTC} \geq 60 \mathrm{~ms}$. Multivariable lineer regresyon was used to assess the relationship of QTc-AT with calsium and potassium level. We constructed two seperate models to assess the relationship of QTC-AT with baseline and 3rd-day potassium and calcium levels after adjusting common clinical and biological plausible predictors ( age, gender, QTC prolonged drug(azitromisin, antiviral teratment), loop diuretic, beta blocker, CHF, CRF, SIRS criteria $\geq 2$, myocardial injury, magnesium). In the first model, the relationship between QTC-AT and baseline calsium, potassium was evaluated. A significant relationship was observed between QTc-AT and baseline potassium $(\beta$ coefficient $=-2.083,95 \% \mathrm{Cl}$ $-27.134--0.577, p$ value $=0.041)$, CHF $(\beta$ coefficient $=$ $2.184,95 \% \mathrm{Cl} 3.529-78.276, \mathrm{p}$ value $=0.032)$, gender(female) ( $\beta$ coefficient $=2.654,95 \% \mathrm{Cl} 3,160$ - 24.598, $p$ value $=0.012)$ and QTC-B ( $\beta$ coefficient $=$
4.818, 95\% Cl 0.279- 0,674, p value $<0,001$ ), and no significant relationship was observed with baseline calcium $(\beta$ coefficient $=-0.848,95 \% \mathrm{Cl}-22.102-$ 8.919, $p$ value $=0,399$ ). (Table 3a) The relationship between QTc-AT and calsium, potassium levels on the 3 rd day were evaluated in the 2 nd model. There was a significant relationship between QTC-AT and potassium level on the 3rd day ( $\beta$ coefficient= $-2.547,95 \% \mathrm{Cl}-27.925--3.385$, $p$ value $=0.013), \mathrm{CHF}$ $(\beta$ coefficient $=2.615,95 \% \mathrm{Cl} 9.199-84.724, p$ value $=$ 0.016 ), SIRS criteria $\geq 2$ ( $\beta$ coefficient $=2.297,95 \% \mathrm{CI}$ 2.247-32.044, $p$ value $=0.025)$, gender (female) $(\beta$ coefficient $=2.874,95 \% \mathrm{Cl} 4.697-26.054$, $p$ value $=$ 0.005 ) and QTC-B ( $\beta$ coefficient $=5.028,95 \% \mathrm{Cl}$ $0.307-0.711, p$ value $<0.001$ ), and no significant relationship was observed with calsium on the $3 \mathrm{rd}$ day $(\beta$ coefficient $=0.707,95 \% \mathrm{Cl}-8.685-20.784, p$ value $=0.415)$ (Table $3 b)$.

Table 2. Laboratory and Electrocardiographic findings of patients

\begin{tabular}{|l|c|}
\hline White blood cell (103/uL) & $6.60(4.78-9.02)$ \\
\hline Neutrophil (103/uL) & $4.25(2.89-7.22)$ \\
\hline Lymphocyte (103/uL) & $1.51(1.12-2.03)$ \\
\hline Plathelet (103/uL) & $232(183-272)$ \\
\hline Hemoglobin g/dL & $13.7(12.3-14.7)$ \\
\hline C-reactive protein mg/L & $13.5(2-50)$ \\
\hline Procalsitonin (ng/mL) & $0.06(0.04-0.14)$ \\
\hline D-dimer (ng/mL) & $175(108-330)$ \\
\hline Ferritin ( $\mathrm{gg} / \mathrm{L})$ & $126(42-33)$ \\
\hline Creatinine (mg/dl) & $0.78(0.67-0.90)$ \\
\hline Sodium (mmol/L) & $138(136-139)$ \\
\hline Magnesium (mg/dL) & $1.94(1.82-2.09)$ \\
\hline Potassium baseline (mmol/L) & $4(3.7-4.3)$ \\
\hline Potassium 3rd day (mmol/L) & $4.2(3.9-4.5)$ \\
\hline Albumin baseline (g/L) & $43(40-45)$ \\
\hline Albumin 3rd day (g/L) & $40(37-43)$ \\
\hline Total calcium baseline (mg/dL) & $8.70(8.40-9.00)$ \\
\hline Total calcium 3rd day (mg/dL) & $8.40(8.10-8.80)$ \\
\hline Corrected calcium baseline (mg/dL) & $8.48(8.24-8.73)$ \\
\hline Corrected calcium 3rd day (mg/dL) & $8.42(8.16-8.70)$ \\
\hline Heart rate baseline (beat/min) & $87(76-99)$ \\
\hline Heart rate 3rd day (beat/min) & $80(74-90)$ \\
\hline QRS duration baseline (ms) & $92(84-102)$ \\
\hline QRS duration after treatment (ms) & $95(88-104)$ \\
\hline QT interval baseline (ms) & $360(334-375)$ \\
\hline QT interval after treatment(ms) & $378(360-400)$ \\
\hline QTc interval baseline (ms) & $427(409-447)$ \\
\hline QTc interval after treatment (ms) & $438(414-459)$ \\
\hline
\end{tabular}

Variables are presented given as median (interquartile range). 
Table 3a. Multivariable and univariable linear regression analysis model I

\begin{tabular}{|c|c|c|c|c|c|c|}
\hline & \multicolumn{3}{|c|}{ Univariable analysis } & \multicolumn{3}{|c|}{ Multivariable analysis } \\
\hline & $\beta$-coefficient & $\mathrm{Cl} 95 \%$ & $P$ value & $\beta$-coefficient & $\mathrm{Cl} 95 \%$ & $P$ value \\
\hline Age & 2.893 & $0.170-0.564$ & 0.004 & 1.969 & $-0.004-0.628$ & 0.053 \\
\hline Gender(female) & 2.864 & $3.888-21.097$ & 0.005 & 2.584 & $3.160-24.598$ & 0.012 \\
\hline $\mathrm{CHF}$ & 2.687 & $8.763-57.206$ & 0.008 & 2.184 & $3.529-78.276$ & 0.032 \\
\hline SIRS criteria $\geq 2$ & 4.283 & $11.862-32.123$ & $<0,001$ & 1.790 & $-1.495-27.530$ & 0.078 \\
\hline Chronic renal failure & 0.574 & $-19.114-34.790$ & 0.567 & -0.358 & $-70.936-49.367$ & 0.722 \\
\hline Myocardial injury & 1.932 & $-0.556-52.855$ & 0.55 & 0.412 & $-31.866-48.444$ & 0.682 \\
\hline Loop diuretic & 1.377 & $-8.102-45.574$ & 0.170 & -1.070 & $-72.681-21.948$ & 0.288 \\
\hline Azithromycin & 0.762 & $-5.471-12.360$ & 0.447 & -0.699 & $-14.630-7.042$ & 0.487 \\
\hline Beta blocker & 2.893 & $7.093-37.532$ & 0.004 & -0.925 & $-32.952-12.082$ & 0.358 \\
\hline Favipiravir & 2.613 & $4.222-30.257$ & 0.01 & -0.653 & $-23.168-11.749$ & 0.516 \\
\hline Magnesium & -0.580 & $-42.105-23.089$ & 0.563 & 0.618 & $-19.273-36.560$ & 0.539 \\
\hline QTc-B & 9.759 & $0.499-0.752$ & $<0.001$ & 4.818 & $0.279-0.674$ & $<0.001$ \\
\hline Calcium baseline & -1.501 & $-21.461-2.915$ & 0.135 & -0.848 & $-22.102-8.919$ & 0.399 \\
\hline Potassium baseline & -1.264 & $-16.752-3.672$ & 0.208 & -2.083 & $-27.134--0.577$ & 0.041 \\
\hline
\end{tabular}

CHF: Congestive Heart Failure; SIRS: Systemic Inflammatory Response Syndrome; QTC-B: QTC baseline; QTc-AT: QTc after treatment; Cl: Confidence Interval

Table 3b. Multivariable and univariable linear regression analysis model II

\begin{tabular}{|l|c|c|c|c|c|c|}
\hline & \multicolumn{3}{|c|}{ Univariable analysis } & \multicolumn{3}{c|}{ Multivariable analysis } \\
\hline & $\beta$-coefficient & Cl 95\% & P value & $\beta$-coefficient & Cl 95\% & P value \\
\hline Age & 2.893 & $0.170-0.564$ & 0.004 & 1,955 & $-0.007-0.623$ & 0.055 \\
\hline Gender(female) & 2.864 & $3.888-21.097$ & 0.005 & 2.874 & $4.697-26.054$ & 0.005 \\
\hline CHF & 2.687 & $8.763-57.206$ & 0.008 & 2.482 & $9.199-84.724$ & 0.016 \\
\hline SIRS criteria $\geq 2$ & 4.283 & $11.862-32.123$ & $<0.001$ & 2.297 & $2.247-32.044$ & 0.025 \\
\hline Chronic Renal Failure & 0.574 & $-19.114-34.790$ & 0.567 & 0.435 & $-50.054-77.990$ & 0.665 \\
\hline Myocardial injury & 1.932 & $-0.556-52.855$ & 0.55 & -0.243 & $-46.417-36.350$ & 0.809 \\
\hline Loop diuretic & 1.377 & $-8.102-45.574$ & 0.170 & -1.648 & $-89.582-8.556$ & 0.104 \\
\hline Azithromycin & 0.762 & $-5.471-12.360$ & 0.447 & -1.044 & $-16.461-5.152$ & 0.300 \\
\hline Beta blocker & 2.893 & $7.093-37.532$ & 0.004 & -1.318 & $-16.461-5.152$ & 0.300 \\
Favipiravir & 2.613 & $4.222-30.257$ & 0.01 & -1.116 & $-25.496-7.211$ & 0.268 \\
\hline Magnesium & -0.580 & $-42.105-23.089$ & 0.563 & 0.816 & $-16.812-40.045$ & 0.418 \\
\hline QTc-B & 9.759 & $0.499-0.752$ & $<0.001$ & 5.028 & $0.307-0.711$ & $<0.001$ \\
\hline Calcium 3rd-day & -0.617 & $-13.717-7.182$ & 0.538 & 0.819 & $-8.685-20.784$ & 0.415 \\
\hline Potassium 3rd-day & -2.885 & $-24.631--4.626$ & 0.004 & -2.547 & $-27.925--3.385$ & 0.013 \\
\hline
\end{tabular}

CHF: Congestive Heart Failure; SIRS: Systemic Inflammatory Response Syndrome; QTc-B: QTC baseline; QTc-AT: QTc after treatment; Cl: Confidence Interval

\section{DISCUSSION}

The main findings of this study are as follows: 1) QTc prolonged significantly in patients who were hospitalized with COVID-19 and started hydroxychloroquine with or without azithromycin therapy. 2) Electrolyte imbalance can be seen in COVID-19 patients, where there was a significant relationship between the QTC-AT interval and baseline potassium and day 3 potassium levels.
However, there was no significant relationship between QTc-AT and calcium levels.

COVID-19 is a disease caused by SARS-COV-2 and although pneumonia is the most common clinical scenario, COVID-19 can affect many organs and tissues. Severe complications such as hypoxemia, acute respiratory distress syndrome, acute cardiac injury, arrhythmias, shock, and acute kidney injury 
have been reported among COVID-19 patients [21, 22]. Electrolyte disorders such as hypocalcemia and hypercalcemia are common in COVID-19 patients, and studies have demonstrated that electrolyte imbalance has a relation with the severity of the disease $[13,14,23]$. In our study, hypokalemia $(<3.5$ $\mathrm{mmol} / \mathrm{L}$ ) was observed in $8.6 \%$ and $53 \%$ of patients had potassium level $\leq 4 \mathrm{mmol} / \mathrm{L}$. Hypocalcemia (corrected calcium $<8.5 \mathrm{mg} / \mathrm{dl}$ ) was observed in $51 \%$ of patients when all calcium values were corrected with albümin whereas hypocalcemia was observed in $24 \%$ of patients when the correction was applied only to those with albumin below $40 \mathrm{mg} / \mathrm{L}$. SARSCoV-2 binds to its host receptor, angiotensinconverting enzyme 2 (ACE2) and likely reduces ACE2 expression, thus leading to increased angiotensin II, which can cause increased potassium excretion by the kidneys, ultimately leading to hypokalemia $[14,15,24]$. The causes of hypocalcemia in critically ill patients can be defined as, vitamin-D deficiency, oversecretion of parathyroid hormone, drug interactions, hypomagnesemia, decreased dietary intake, hypoproteinemia, and so on [25]. The mechanism of hypocalcemia in COVID-19 patients remains a subject in need of clarification.

The QT interval reflects the duration of ventricular electrical activity and the prolonged QTC interval was associated with arrhythmias and a high mortality rate [8-10]. There are many causes of QTc prolongation, and one of them is drugs. Hydroxychloroquine, azithromycin, and some antiviral drugs used in COVID-19 treatment are known to prolong QTc interval [5-7]. In a recent study by Nikolas J. et al. it was revealed that in COVID-19 patients, a significant prolongation in the QTc interval was detected with the use of hydroxychloroquine with or without azithromycin, and torsades de pointes were observed in one patient [26]. Similarly, in our study, a significant prolongation was observed in the QTc interval, but torsades de pointes was not observed. It is known that electrolyte abnormalities such as hypocalcemia and hypokalemia prolong the QTC interval. Hypocalcemia is a well-known cause of QT prolongation via prolongation of the plateau phase of the cardiac action potential [27, 28]. The electrophysiological effects of hypokalemia include resting membrane hyperpolarization, $\mathrm{Na}+-\mathrm{K}+$ ATPase inhibition, and suppression of $\mathrm{K}+$ channel conductances resulting in action potential duration prolongation [29]. As far as we know there has been no previous study investigating the effect of electrolyte abnormalities on the QTc interval in COVID-19 patients. In our study, a significant relationship was observed between QTC-AT and potassium level of baseline and the 3rd day of hospitalization, and the patients whose potassium level was lower had a longer QTc interval. There was no significant relationship between QTc-AT and baseline calcium levels and calcium levels on the 3rd day. The physiologically active part of total calcium is ionized calcium. Hypocalcemia is common due to protein abnormalities secondary to an infection, and hypocalcemia may not affect QTc due to normal ionized calcium levels [30].

Patients admitted with COVID-19 have a longer basal QTc interval and a higher risk of potential arrhythmia due to the metabolic and physiological effects of the disease and comorbidities [31]. A risk score for drug-associated QTc prolongation has been derived and validated by Tisdale et al. for the prediction of drug-associated QT prolongation among cardiac care unit-hospitalized patients. In that study, independent predictors of QTC prolongation included the following: female, diagnosis of myocardial infarction, serum $\mathrm{K}^{+}<3.5$ $\mathrm{mmol} / \mathrm{L}$, left ventricular dysfunction, age $>68$ years, administration of a QT-prolonging drug, $\geq 2$ QT-prolonging drugs or loop diuretic, septic shock and admitting QTC $>450$ ms [16]. In a recent study examining the effect of hydroxychloroquine with or without azithromycin treatment on the QTc interval in COVID-19 patients, having two or more SIRS criteria was found to be independently associated with QTc prolongation [26]. In our study, there was also a significant relationship between QTC-AT interval and gender(female), CHF, QTc-B interval in the first model. In the second model, there was a significant relationship between QTC-AT and CHF, SIRS criteria $\geq 2$, QTc-B, and gender (female).

In the guidance published by the European Society of Cardiology on the management of COVID-19 patients, recommendations were made regarding the management of QTC in patients taking drugs that prolong QTc [32]. If the patient has a QTC $\geq$ $500 \mathrm{~ms}$ or shows a $\Delta \mathrm{QTc} \geq 60 \mathrm{~ms}$, consideration should be given to either switching to a drug with a lower risk of QTc prolongation, reducing the dose administered, or continuing the treatment plan. It has also been suggested to keep potassium levels above $4.5 \mathrm{mmol} / \mathrm{L}$ in these patient groups. İn our 
study after treatment, 11 patients had QTC $\geq 500$ $\mathrm{ms}$ and/or $\triangle \mathrm{QTC} \geq 60 \mathrm{~ms}$. It was determined that azithromycin or hydroxychloroquine treatment was discontinued in 6 patients due to prolongation of QTc interval. In 7 of these 11 patients, potassium levels $<4 \mathrm{mmol} / \mathrm{L}$ was observed on the 3rd day and it was observed that only 2 patients had potassium replacement.

Considering the significant relationship between hypokalemia and QTc prolongation, close monitoring of potassium levels and QTc interval is important in this patient group, where electrolyte imbalance such as hypokalemia can be seen. Besides, in patients with QTc prolongation and hypokalemia, the risk of arrhythmia can be reduced with potassium replacement, and drugs that prolong QTC such as hydroxychloroquine and azithromycin may continue.

\section{Limitations}

Our study had some limitations. The most important limitation of the study is the small number of patients and larger studies are needed on the relationship between electrolyte imbalance and QTC interval in patients with COVID-19. The other limitation of the study the use of the same magnesium levels in both regression models due to a one-time measurement. In addition, corrected calcium levels were evaluated in the study because ionized calcium, the active form of serum calcium, was not measured on admission and 3rd-day levels in most patients. lonized calcium levels could be more closely related to the QTc interval.

\section{CONCLUSION}

Electrolyte disorders such as hypocalcemia and hypokalemia can be seen in patients hospitalized with COVID-19. After treatment, a significant prolongation was observed in the QTc interval and a significant relationship was observed between QTc-AT and potassium levels, but no significant relationship with calcium was observed.

\section{CONFLICTS Of INTERESTS}

The authors declared no conflicts of interest. 
[1] Wu Z, McGoogan JM. Characteristics of and important lessons from the coronavirus disease 2019 (COVID-19) outbreak in China: Summary of a report of 72314 cases from the Chinese Center for Disease Control and Prevention. JAMA 2020; 323: 1239-1242.

[2] World Health Organization. WHO Director-General's opening remarks at the media briefing on COVID-19-11 March 2020. https://www.who.int/dg/speeches/detail/ who-directorgeneral-s-opening-remarks-at-the-mediabriefing-on-covid19-11-march-2020. Accessed March 24, 2020.

[3] World Health Organization. Statement on the second meeting of the International Health Regulations (2005) Emergency Committee regarding the outbreak of novel coronavirus (2019-nCoV). https://www.who. $\mathrm{int} /$ news-room/detail/30-01-2020-statement-on-thesecond-meeting-of-theinternational-health-regulations(2005)-emergency-committee-regarding-the-outbreakof-novel-coronavirus-(2019-ncov). Accessed March 24, 2020.

[4] Madjid M, Safavi-Naeini P, Solomon SD, et al. Potential Effects of Coronaviruses on the Cardiovascular System: A Review. JAMA Cardiol. Published online March 27, 2020. doi:10.1001/jamacardio.2020.1286

[5] Straus SM, Kors JA, De Bruin ML, et al. Prolonged QTC interval and risk of sudden cardiac death in a population of older adults. J Am Coll Cardiol. 2006; 47:362-7.

[6] Zhang Y, Post WS, Dalal D, et al. QT-interval duration and mortality rate: results from the Third National Health and Nutrition Examination Survey. Arch Intern Med. 2011; 171: 1727-33.

[7] Beinart R, Zhang Y, Lima J, et al. The QT interval is associated with incident cardiovascular events: the MESA study. J Am Coll Cardiol. 2014; 64: 2111-9.

[8] Chorin E, Wadhwani L, Magnani S, et al. QT Interval Prolongation and Torsade De Pointes in Patients with COVID-19 treated with Hydroxychloroquine/Azithromycin [published online ahead of print, 2020 May 11]. Heart Rhythm. 2020; S1547-5271(20)30435-5.

[9] World Health Organization. The cardiotoxicity of antimalarials: Malaria Policy Advisory Committee Meeting. Published March 24, 2017. Accessed April 21, 2020. https://www.who.int/malaria/mpac/ mpac-mar2017-ergcardiotoxicity-report-session2.pdf

[10] Ray WA, Murray KT, Hall et al. Azithromycin and the risk of cardiovascular death. N Engl J Med. 2012; 366(20): 18811890.

[11] Guan W-J, Ni Z-Y, Hu Y, et al. Clinical characteristics of coronavirus disease 2019 in China. N Engl J Med. 2020; 382: 1708-1720.

[12] Huang C, Wang Y, Li X, et al. Clinical features of patients infected with 2019 novel coronavirus in Wuhan, China. Lancet. 2020; 395: 497-506.
[13] Lippi G, South AM, Henry BM. Electrolyte imbalances in patients with severe coronavirus disease 2019 (COVID-19). Ann Clin Biochem. 2020; 57(3): 262-265

[14] Chen, D, Li, X, Song, Q, et al. Hypokalemia and clinical implications in patients with coronavirus disease 2019 (COVID-19). medRxiv. Epub ahead of print 29 February 2020. doi:10.1101/2020.02.27.20028530

[15] El-Sherif N, Turitto G. Electrolyte disorders and arrhythmogenesis. Cardiol J. 2011; 18(3): 233-245.

[16] Tisdale JE, Jayes HA, Kingery JR, et al. Development and validation of a risk score to predict QT interval prolongation in hospitalized patients. Circ Cardiovasc Qual Outcomes. 2013; 6: 479-487.

[17] Gulhar R, Jialal I. Physiology, Acute Phase Reactants. [Updated 2020 Mar 20]. In: StatPearls [Internet]. Treasure Island (FL): StatPearls Publishing; 2020 Jan. Available from: https://www.ncbi.nlm.nih.gov/books/NBK519570/

[18] Payne RB, Little AJ, Williams RB, et al. Interpretation of serum calcium in patients with abnormal serum proteins. Br Med J. 1973; 4(5893): 643-646.

[19] Liamis G, Milionis HJ, Elisaf M. A review of drug-induced hypocalcemia. J Bone Miner Metab. 2009; 27(6): 635-642.

[20] Kardalas E, Paschou SA, Anagnostis P, et al. Hypokalemia: a clinical update. Endocr Connect. 2018; 7(4): R135-R146.

[21] Huang C, Wang Y, Li X, Ren L, et al. Clinical features of patients infected with 2019 novel coronavirus in Wuhan, China. Lancet 2020.

[22] Chen N, Zhou M, Dong X, et al. Epidemiological and clinical characteristics of 99 cases of 2019 novel coronavirus pneumonia in Wuhan, China: a descriptive study. Lancet. 2020; 395: 507-13.

[23] Min Cao, Dandan Zhang, Youhua Wang, et al. ; Clinical Features of Patients Infected with the 2019 Novel Coronavirus (COVID-19) in Shanghai, China medRxiv 2020.03.04.20030395

[24] Liu, Y, Yang, Y, Zhang, C, et al. : Clinical and biochemical indexes from 2019-nCoV infected patients linked to viral loads and lung injury. Sci China Life Sci. 2020; 63: 364-374.

[25] Kelly A, Levine MA. Hypocalcemia in the critically ill patient. J Intensive Care Med. 2013; 28(3): 166-177.

[26] Mercuro NJ, Yen CF, Shim DJ, et al. ; Risk of QT Interval Prolongation Associated With Use of Hydroxychloroquine With or Without Concomitant Azithromycin Among Hospitalized Patients Testing Positive for Coronavirus Disease 2019 (COVID-19) [published online ahead of print, 2020 May 1]. JAMA Cardiol. 2020; e201834. doi:10.1001/ jamacardio.2020.1834

[27] Eryol NK, Colak R, Ozdogru I, et al. ; Effects of calcium treatment on QT interval and QT dispersion in hypocalcemia. Am J Cardiol. 2003; 91: 750-2.

[28] Bronsky D, Dubin A, Waldstein SS, et al. Calcium and the electrocardiogram. I. The electrocardiographic manifestations of hypoparathyroidism. Am J Cardiol. 1961; 7: 823-32 
[29] Weiss JN, Qu Z, Shivkumar K. Electrophysiology of Hypokalemia and Hyperkalemia. Circ Arrhythm Electrophysiol. 2017; 10(3): e004667. doi:10.1161/ CIRCEP.116.004667

[30] Keihani Douste Z, Haghi Ashtiani MT, Shariat M, et al. Comparative Evaluation of Corrected QT and lonized Calcium in Children. Acta Med Iran. 2015; 53(9): 579-581.

[31] Simpson TF, Kovacs RJ, Stecker EC. Ventricular arrhythmia risk due to hydroxychloroquine-azithromycin treatment for COVID-19. March 29, 2020. Available at: https://www. acc.org/latest-in-cardiology/articles/2020/03/27/14/00/ ventriculararrhythmia-risk-due-to-hydroxychloroquineazithromycin-treatment-for-covid-19

[32] The European Society for Cardiology. ESC Guidance for the Diagnosis and Management of CV Disease during the COVID-19 Pandemic. https://www.escardio.org/ Education/COVID-19-and-Cardiology/ESC-COVID-19Guidance. (Last update: 28 May 2020) 